\title{
Manejo ortodóncico de la clase III: presentación de un caso clínico.
}

\section{Orthodontic management class III: presentation of a clinical case.}

\author{
Cynthia Marlen Horta Sánchez,* Fernando Parés Vidrio ${ }^{\ddagger}$
}

\section{RESUMEN}

Introducción: La maloclusión clase III es considerada como severa y en la mayoría de los pacientes la etiología suele estar combinada entre componentes esqueléticos y dentoalveolares. Las posibilidades terapéuticas dependerán de la edad biológica del paciente y del tipo de maloclusión. Conforme va aumentando la edad del paciente va menguando la capacidad de crecimiento y se va asentando la relación de clase III esquelética. Las maloclusiones de clase III esqueléticas se pueden corregir mediante extracciones dentales y cirugía ortognática. Caso clínico: Paciente masculino de 13 años de edad con protrusión mandibular, discrepancia óseo dentaria negativa, convexidad facial disminuida y clase III dental y esqueletal. Resultados: Se resolvió la discrepancia óseo dentaria negativa del paciente, se logró la clase I Molar y canina con una sobremordida adecuada.

Palabras clave: Maloclusión, Angle, clase III.

\section{ABSTRACT}

Introduction: Class III malocclusion is considered severe and in most patients the etiology is usually combined between skeletal and dentoalveolar components. The therapeutic possibilities depend on the biological age of the patient and the type of malocclusion. As it increases the age of the patient wanes growth capacity and Will settled the relationship skeletal class III. The skeletal class III malocclusion can be corrected by tooth extractions and orthognathic surgery. Case report: Male patient 13 years old with mandibular protrusion, negative tooth bone discrepancy, decreased facial convexity and dental and skeletal class III. Results: Dental patient refusal bone discrepancy was resolved, I molar and canine class and adequate overbite was achieved.

Keywords: Malocclusion, Angle, class III.

\section{INTRODUCCIÓN}

$\mathrm{L}$ a clase III se define cuando el surco vestibular del primer $ـ$ molar inferior permanente está por mesial de la cúspide mesiovestibular del primer molar superior permanente. ${ }^{1}$

La relación clase III es la menos prevalente de las tres clasificaciones de Angle, existe una variación geográfica según el grupo racial y étnico. Esta relación oclusal es particularmente común en individuos con ancestros asiáticos, encontrándose en la población China en un porcentaje de $12 \%$, y es menor su aparición en europeos (1.5 a 5.3\%) y norteamericanos caucásicos (1 a 4\%). ${ }^{2,3}$

\footnotetext{
* Estudiante de la Especialidad en Estomatología en el Niño y el Adolescente.

¥ Maestro en Ciencias Odontológicas, Especialista en Ortodoncia y Profesor titular de la Especialidad en el Niño y el Adolescente.

Universidad Nacional Autónoma de México.

Recibido: 17 Septiembre 2019.

Aceptado para publicación: 03 Diciembre 2019.
}

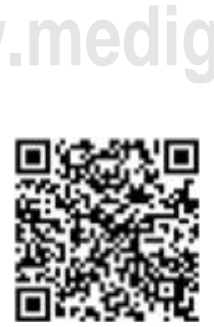

La clase III se clasifica en:

- Dentoalveolar: por una retrusión dentoalveolar maxilar o una protrusión dentoalveolar mandibular.

- Esquelética: por una deficiencia maxilar, un exceso mandibular y/o ambas.

En la etiología de la maloclusión clase III se conjugan factores hereditarios y factores medioambientales como hábitos, respiración oral y patrones alterados de erupción entre otros. ${ }^{4}$

Entre los factores dentoalveolares involucrados se destacan las alteraciones en tiempo y patrones de erupción de los dientes anteriores, que favorecen el desarrollo de una mordida cruzada anterior de tipo dental. ${ }^{5}$

Los pacientes con maloclusión clase III pueden presentar combinación de condiciones esqueletales y dentoalveolares. ${ }^{6}$

Algunas de las características clínicas comunes de la maloclusión clase III son: ${ }^{7}$ 
1. Perfil cóncavo.

2. Deficiencia en el crecimiento anteroposterior del recio medio facial, que a menudo involucra al maxilar y al cigomático o malar.

3. Deficiencia transversal.

4. Exceso mandibular originado por una longitud incrementada por un posicionamiento anterior de la cavidad glenoidea, lo que genera un posicionamiento anterior de la mandíbula.

5. Aumento de la altura facial anterior inferior con patrón de crecimiento vertical.

6. Mordida cruzada o borde a borde anterior.

7. Mordida cruzada posterior.

8. Apiñamiento leve, moderado o severo en el arco superior.

9. Proinclinación de los incisivos superiores.

10. Retroinclinación de los incisivos inferiores.
Las características cefalométricas más comunes son: ${ }^{8}$

a) Base del cráneo anterior (S-Na) corta.

b) Ángulo de la base del cráneo (Ba-S-Na) cerrado.

c) Longitud maxilar (ENA-ENP) disminuida.

d) Punto A posicionado posteriormente.

e) Longitud del cuerpo mandibular (Go-Gn) aumentada.

f) Longitud disminuida de la rama mandibular.

g) Ángulo mandibular (Ar-Go-Me) abierto.

h) Ángulo del plano mandibular aumentado.

Es importante resaltar los objetivos de tratar de manera temprana la maloclusión clase III:

1. Prevenir cambios irreversibles en tejidos blandos y en el hueso. La mordida cruzada anterior sin tratar puede ocasionar desgaste anormal de incisivos, adelgazamiento de tablas óseas y recesiones gingivales. ${ }^{9}$
Figura 1:

Fotografías extraorales iniciales. A) Fotografía de frente. B) Fotografía de perfil donde se observa un perfil cóncavo.
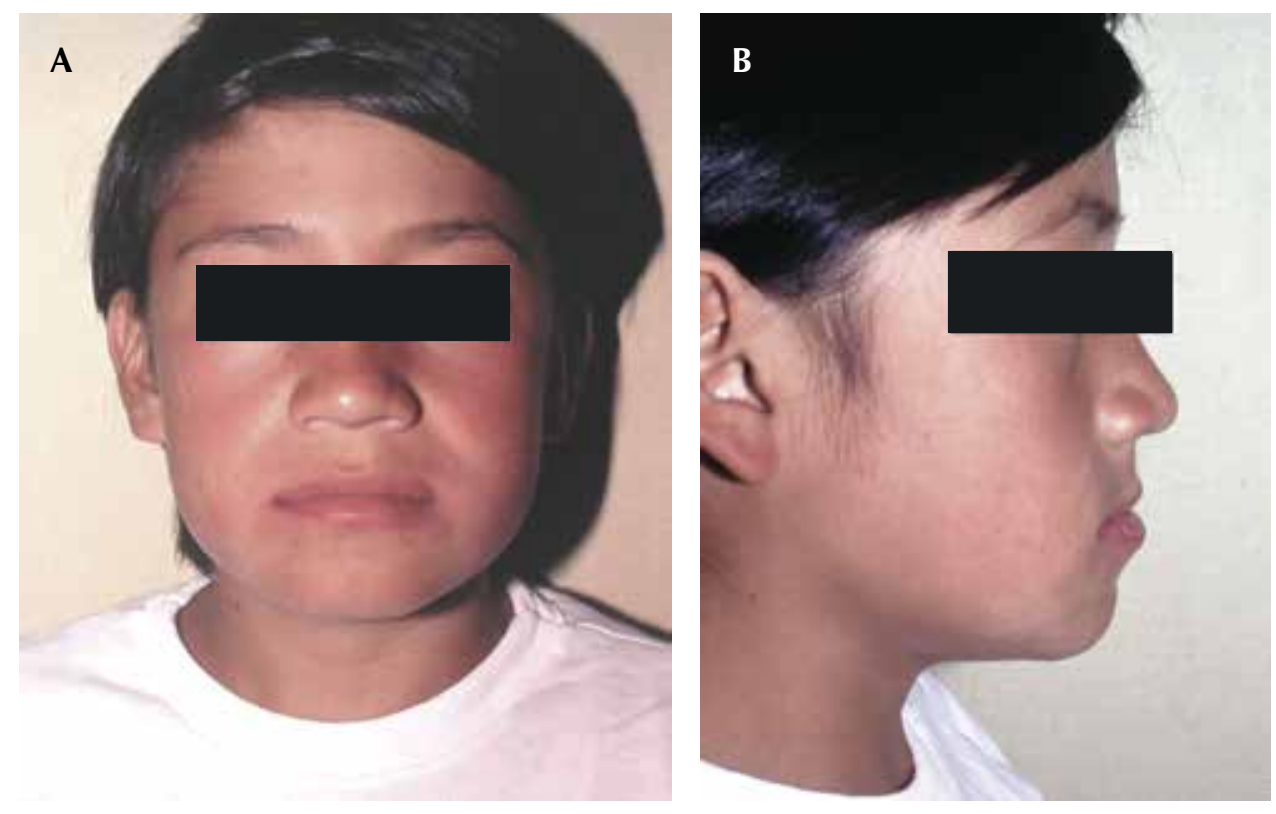
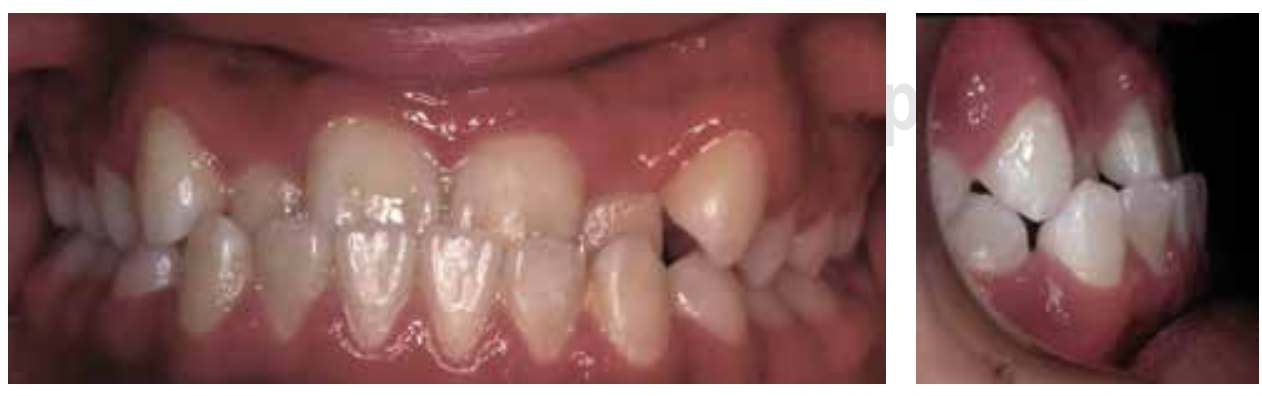

Figura 2:

Fotografías intraorales iniciales donde se observa la mordida cruzada anterior. 
Figura 3:

Fotografías intraorales de la arcada superior e inferior, donde se observa la discrepancia óseodentaria negativa.
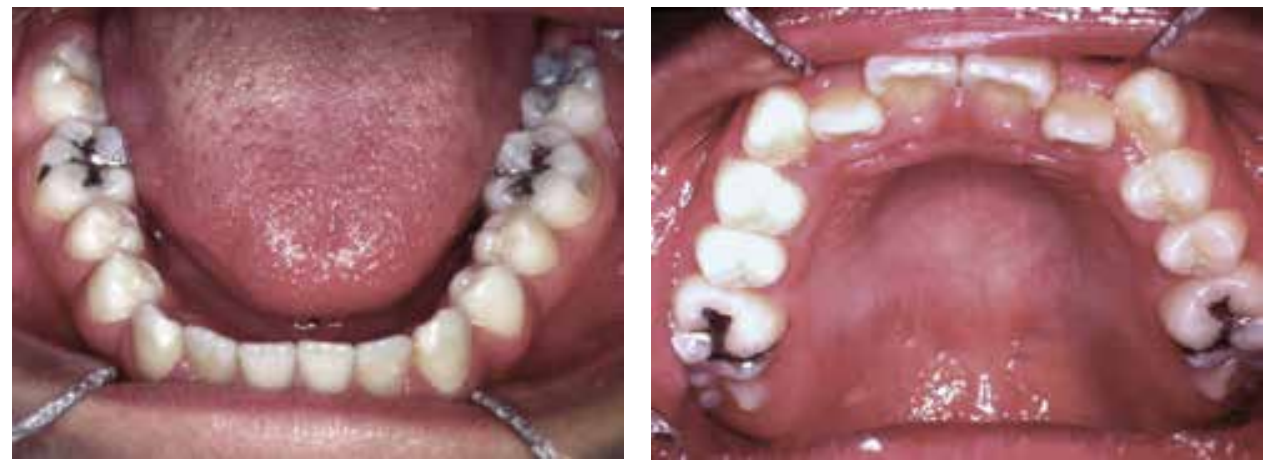

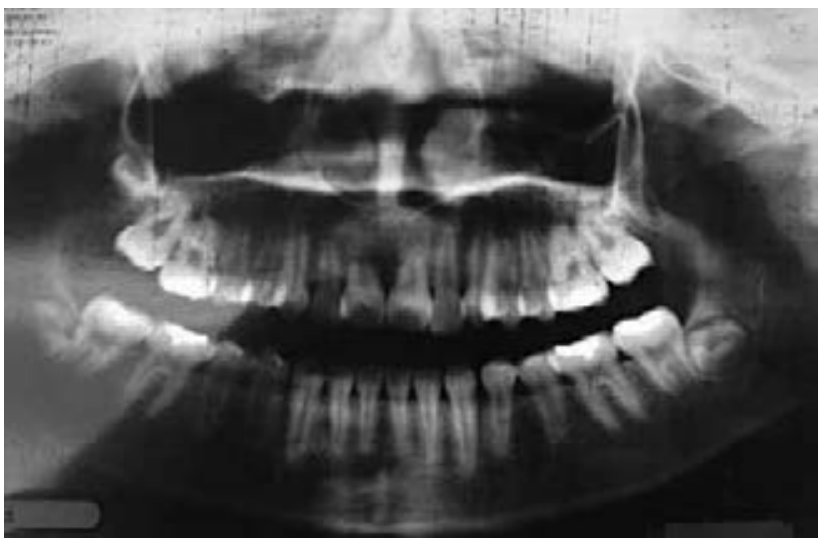

Figura 4: Se observa la radiografía panorámica donde se pueden notar las raíces enanas de los incisivos centrales superiores.

2. Mejorar la función oclusal eliminando discrepancias entre oclusión céntrica y relación céntrica. ${ }^{9}$

3. En maloclusiones leves o moderadas, puede eliminarse la necesidad de cirugía ortognática si se realiza un tratamiento ortopédico temprano y, en casos más complejos, puede disminuirse la extensión de los procedimientos quirúrgicos. ${ }^{9}$

4. El tratamiento temprano mejora las condiciones estéticas y asimismo la autoestima de los niños; el manejo oportuno de la maloclusión clase III beneficia la apariencia facial y la postura labial. ${ }^{9}$

Las posibilidades terapéuticas dependerán de la edad biológica del paciente y del tipo de maloclusión. Si el paciente presenta una maloclusión dentoalveolar quizá sólo sea necesario tratarlo con placas activas; sin embargo, si es esquelética tendrá que tratarse con ortopedia, dado que si ya han erupcionado los dientes permanentes disminuye la probabilidad de tratarlo con ortopedia y se puede considerar la cirugía ortognática como una opción de tratamiento. ${ }^{10}$

\section{PRESENTACIÓN DEL CASO}

Sumario del diagnóstico (Figuras 1 a 5):

1. Paciente masculino de 13 años de edad en crecimiento, presenta una maloclusión clase III.

2. Discrepancia óseo dentaria negativa.

3. Incisivos superiores retrusivos e incisivos inferiores protruidos.

4. Mordida cruzada anterior.

5. Raíces enanas en los incisivos centrales superiores.

6. Convexidad facial disminuida.

7. Retrusión maxilar.

8. Protrusión.

\section{Objetivos del tratamiento}

I. Corregir la maloclusión y la relación esquelética.

II. Eliminar la discrepancia óseo-dentaria superior e inferior.

III. Eliminar la mordida cruzada anterior.

IV. No agravar las raíces enanas en los incisivos centrales superiores

V. Conseguir clase I molar y canina.

VI. Coordinar los arcos maxilares.

VII. Mejorar líneas medias.

VIII. Mejorar el perfil facial.

\section{Plan de tratamiento}

El tratamiento consistió en primero realizar expansión con Hyrax y colocación de bite block ${ }^{\S}$ para descruzar

\footnotetext{
$\S$ Nota del editor: Un bite block (bloque de mordida) es un aditamento dental, se usan para ayudar a mantener la boca y la mandíbula del paciente lo suficientemente abiertas durante un procedimiento ortodóncico. Este pequeño accesorio bucal está diseñado para proporcionar suficiente fuerza para evitar que el paciente cierre, siendo lo suficientemente suave como para proporcionarle comodidad.
} 
Figura 5:

Se observa la radiografía lateral de cráneo y el trazado cefalométrico de Ricketts iniciales.
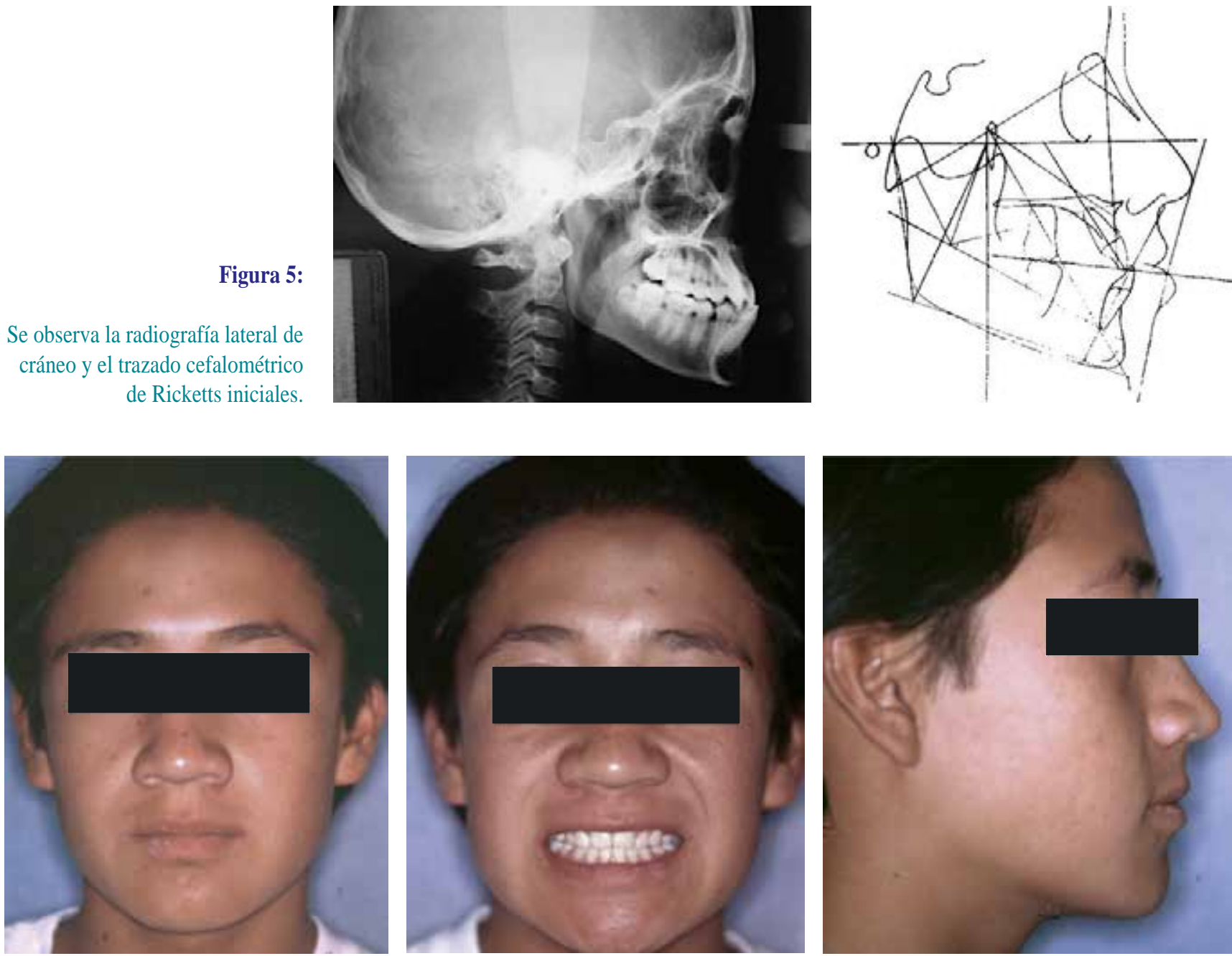

Figura 6: Fotografías extraorales finales donde se observa la mejoría que se logró en el perfil del paciente.

la mordida anterior; protracción maxilar con máscara facial durante un año. Posteriormente colocación de aparatología fija con previa extracción de primeros premolares superiores e inferiores. Utilización de arcos de baja fricción y fuerzas ligeras debido a las raíces enanas de los incisivos centrales superiores. Se utilizaron arcos de retrusión para cierre de espacios, arcos y elásticos para finalización.

\section{RESULTADOS}

Se logró descruzar la mordida anterior, aliviar la discrepancia óseo-dentaria superior e inferior. También se logró conseguir una clase I molar y canina en ambos arcos maxilares. Preservar en buen estado las raíces

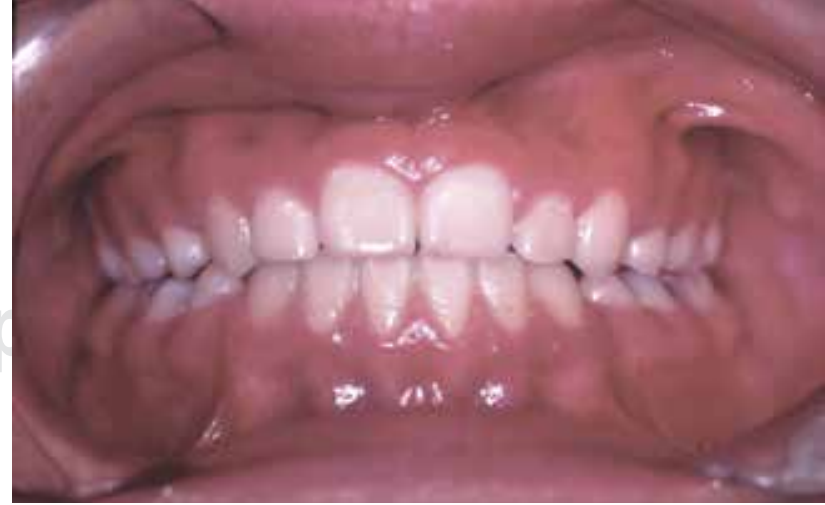

Figura 7: Fotografía intraoral frontal final, donde se puede observar la eliminación de la mordida cruzada anterior, líneas medias alineadas. 
Figura 8:

Fotografías laterales finales: se puede observar adecuada intercuspidación y clase I molar y canina.
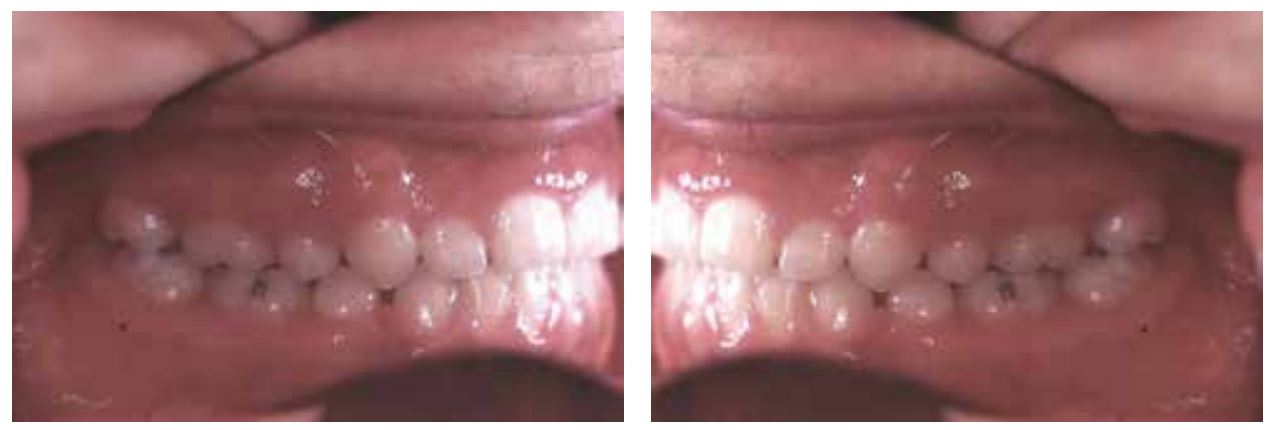

enanas de los incisivos centrales superiores. Asimismo, se logró mejorar el perfil facial y la estética de nuestro paciente (Figuras 6 a 8).

\section{DISCUSIÓN}

De acuerdo a la información revisada, varios autores refieren que este tipo de casos se aproximan generalmente mediante dos alternativas. Una de ellas sería el tratamiento interdisciplinario, el cual contempla ortodoncia y cirugía ortognática, y otro sería encaminado a la ortopedia y a la compensación dentoalveolar. Esta última es la opción que se determinó escoger y con la cual se consiguió resolver la discrepancia dentoalveolar, un mejor equilibrio esquelético y una estética facial con estabilidad al paciente, objetivos que se plantearon al inicio del tratamiento.

\section{CONCLUSIONES}

a) Se puede manejar adecuadamente un paciente clase III realizando protracción maxilar con máscara facial y colocando un bite block para descruzar la mordida.

b) Para preservar las raíces enanas en un tratamiento de ortodoncia es indispensable realizar el tratamiento con fuerzas y arcos ligeros.

\section{BIBLIOGRAFÍA}

1. Quintero Y. Relación esquelética clase III con factor genético predominante. Reporte de un caso. Rev CES Odon. 2007; 20 (2): 44-50.

2. Moyers R. Manual de Ortodoncia, Panamericana, Buenos Aires, 4⿳亠口了 ed. 1992.

3. Quirós ÁO. Manual de Ortopedia Funcional de los Maxilares y Ortodoncia Interceptiva, Caracas, Venez.:Actualidades Médico Odontológicas Latinoamérica, 1995.

4. Avalos GG, Paz CA. Maloclusión clase III revisión bibliográfica. Revista Tamé. 2014; 3 (8): 279-282.

5. Temelcea A, Simion I, Stanciu R, Stanciu D. Intervention of the occlusion relations in the appearance of the modifications in sagittal and transversal plan in the anomalies of the class III angle. J Med Life. 2010; 3 (3): 239-241.

6. Cutovic T. Cephalometric analysis of the middle part of the face in patients with mandibular prognathism. Vojnosanit Pregl. 2014; 71 (11): 1026-1033.

7. Uribe RG. Cárdenas JD. Temprano no, a tiempo. Fondo editorial CIB: Medellin, Colombia; 2014. pp. 251-260.

8. Steiner CC. Cepaholometrics in clinical practice. Angle Orthod. 1959; 29: 8-29.

9. Graber T, Rakosi T, Petrovic A. Ortopedia dentofacial con aparatos funcionales. 2da. edición: Mosby; 1997.

10. Hernández J. La máscara facial de protracción en el tratamiento temprano de la maloclusión clase III. Revista Estomatología. 2006; 14 (2): 6-11.

Correspondencia:

Parés Vidrio Fernando

E-mail: paresfer@yahoo.es 